\title{
Research Article \\ Existence of Solutions of a Discrete Fourth-Order Boundary Value Problem
}

\author{
Ruyun Ma, Chenghua Gao, and Yongkui Chang \\ Department of Mathematics, Northwest Normal University, Lanzhou 730070, China \\ Correspondence should be addressed to Ruyun Ma, ruyun_ma@126.com \\ Received 4 December 2009; Revised 30 January 2010; Accepted 25 March 2010 \\ Academic Editor: Leonid Shaikhet
}

Copyright (C) 2010 Ruyun Ma et al. This is an open access article distributed under the Creative Commons Attribution License, which permits unrestricted use, distribution, and reproduction in any medium, provided the original work is properly cited.

Let $a, b$ be two integers with $b-a \geq 5$ and let $\mathbb{T}_{2}=\{a+2, a+3, \ldots, b-2\}$. We show the existence of solutions for nonlinear fourth-order discrete boundary value problem $\Delta^{4} u(t-2)=f(t, u(t)$, $\left.\Delta^{2} u(t-1)\right), t \in \mathbb{T}_{2}, u(a+1)=u(b-1)=\Delta^{2} u(a)=\Delta^{2} u(b-2)=0$ under a nonresonance condition involving two-parameter linear eigenvalue problem. We also study the existence and multiplicity of solutions of nonlinear perturbation of a resonant linear problem.

\section{Introduction}

The deformations of an elastic beam whose both ends are simply supported are described by a fourth-order two-point boundary value problem

$$
\begin{aligned}
& y^{\prime \prime \prime \prime}+g(x) y=e(x), \quad 0<x<1, \\
& y(0)=y(1)=y^{\prime \prime}(0)=y^{\prime \prime}(1)=0
\end{aligned}
$$

See studies by Aftabizadeh [1] and Gupta in [2]. The existence of solutions of nonlinear boundary value problems of fourth-order differential equations has been studied by many authors; see [1-12] and the references therein. For example, Aftabizadeh [1] proved an existence theorem for nonlinear boundary value problems

$$
\begin{gathered}
y^{\prime \prime \prime \prime}=f\left(x, y, y^{\prime \prime}\right), \quad 0<x<1, \\
y(0)=y_{0}, \quad y^{\prime \prime}(0)=y_{1}, \quad y(1)=\bar{y}_{0}, \quad y^{\prime \prime}(1)=\bar{y}_{1},
\end{gathered}
$$


under several conditions that $f$ is a bounded function. Yang [3] obtained existence results of (1.2) under the following assumption.

(A) There are constants $a, b, c \geq 0$ with $a / \pi^{4}+b / \pi^{2}<1$ such that

$$
|f(x, y, u)| \leq a|y|+b|u|+c
$$

Del Pino and Manásevich [4] extended Yang's result and proved the following.

Theorem A. Assume that the pair $(\alpha, \beta)$ satisfies

$$
\frac{\alpha}{(k \pi)^{4}}+\frac{\beta}{(k \pi)^{2}} \neq 1
$$

for all $k \in \mathbb{N}$ and that there are positive constants $a, b$, and $c$ such that

$$
\begin{gathered}
a \max _{k \in \mathbb{N}}\left\{\frac{1}{\left|k^{4} \pi^{4}-\alpha-\beta k^{2} \pi^{2}\right|}\right\}+b \max _{k \in \mathbb{N}}\left\{\frac{\lambda_{k}}{\left|k^{4} \pi^{4}-\alpha-\beta k^{2} \pi^{2}\right|}\right\}<1, \\
|f(t, u, v)-(\alpha u-\beta v)| \leq a|u|+b|v|+c
\end{gathered}
$$

for all $x \in(0,1), u, v \in \mathbb{R}$, then (1.2) possesses at least one solution.

Of course, the natural question is whether or not the similar existence can be established for the corresponding discrete analog of (1.2) of the form

$$
\begin{gathered}
\Delta^{4} u(t-2)=f\left(t, u(t), \Delta^{2} u(t-1)\right), \quad t \in \mathbb{T}_{2}, \\
u(a+1)=r_{1}, \quad u(b-1)=r_{2}, \quad \Delta^{2} u(a)=r_{3}, \quad \Delta^{2} u(b-2)=r_{4},
\end{gathered}
$$

where $\mathbb{T}_{2}=\{a+2, a+3, \ldots, b-2\}, r_{i} \in \mathbb{R}$ for $i \in\{1,2,3,4\}$.

The purpose of this paper is to show that the answer is yes. To this end, we state and prove a spectrum result of two-parameter linear eigenvalue problem

$$
\begin{aligned}
& \Delta^{4} u(t-2)+\beta \Delta^{2} u(t-1)-\alpha u(t)=0, \quad t \in \mathbb{T}_{2} \\
& u(a+1)=u(b-1)=\Delta^{2} u(a)=\Delta^{2} u(b-2)=0 .
\end{aligned}
$$

This result is a slightly generalized version of Shi and Wang [13, Theorem 2.1]. In Section 3, we use Leray-Schauder principle to study the existence of solutions of (1.6), (1.7) under some nonresonant conditions involving the spectrum of (1.8), (1.9). Section 4 is considered with some perturbations of resonant linear problems. We established some a priori bounds and used these together with bifurcation arguments to prove the existence and multiplicity of solutions.

Finally, we note that the existence of solutions of second-order discrete boundary value problems has also received much attention; see studies by Agarwal and Wong in [14], 
Henderson in [15], and the references therein. However, relatively little is known about the existence of solutions of fourth-order discrete boundary value problems. The likely reason may be that the structure of spectrum of the corresponding linear eigenvalue problem is not very clear. To our best knowledge, only He and Yu [16] as well as Zhang et al. [17] dealt with the discrete problem of the form

$$
\begin{gathered}
\Delta^{4} u(t-2)=f(t, u(t)), \quad t \in \mathbb{T}_{2}, \\
u(a)=u(b)=\Delta^{2} u(a)=\Delta^{2} u(b-2)=0 .
\end{gathered}
$$

As we will see in Section 2, (1.9) has more advantage than (1.11) in the study of the spectrum of two-parameter linear eigenvalue problems.

\section{Spectrum of Two-Parameter Linear Eigenvalue Problem}

Let $a, b$ be two integers with $b-a \geq 5$. Recall

$$
\mathbb{T}_{2}=\{a+2, a+3, \ldots, b-2\}
$$

Let

$$
\begin{gathered}
\mathbb{T}_{0}=\{a, a+1, \ldots, b\}, \quad \mathbb{T}_{1}=\{a+1, a+2, \ldots, b-1\}, \\
\Lambda=\{1,2, \ldots, b-a-3\}
\end{gathered}
$$

Let $X$ be the Banach space

$$
X=\left\{u \mid u: \mathbb{T}_{0} \longrightarrow \mathbb{R}, u(a+1)=u(b-1)=\Delta^{2} u(a)=\Delta^{2} u(b-2)=0\right\}
$$

under the norm

$$
\|u\|_{X}:=\max \left\{|u(j)| \mid j \in \mathbb{T}_{2}\right\}
$$

Let $Y$ be the Banach space

$$
Y=\left\{y \mid y: \mathbb{T}_{1} \longrightarrow \mathbb{R}, y(a+1)=y(b-1)=0\right\}
$$

equipped with the norm

$$
\|y\|_{Y}:=\max \left\{|y(j)| \mid j \in \mathbb{T}_{2}\right\}
$$

Let $Z$ be the Banach space

$$
Z=\left\{z \mid z: \mathbb{T}_{2} \longrightarrow \mathbb{R}\right\}
$$


equipped with the norm

$$
\|z\|_{Z}:=\max \left\{|z(j)| \mid j \in \mathbb{T}_{2}\right\}
$$

Remark 2.1. For any $z \in Z$ with

$$
z=\{z(a+2), z(a+3), \ldots, z(b-2)\}
$$

it determines a unique element $y \in Y$ by

$$
y=\{0, z(a+2), z(a+3), \ldots, z(b-2), 0\}
$$

and a unique element $x \in X$ by

$$
x=\{-z(a+2), 0, z(a+2), z(a+3), \ldots, z(b-2), 0,-z(b-2)\} .
$$

Hence, the Banach spaces $X, Y$, and $Z$ are homomorphic with each other. Denote the natural homomorphism from $Z$ to $X$ by $j$.

Now, we define a linear operator $L: X \rightarrow Z$ by

$$
L u(t):=\Delta^{4} u(t-2)+\beta \Delta^{2} u(t-1)-\alpha u(t), \quad t \in \mathbb{T}_{2}
$$

For $k \in \Lambda$, let $\lambda_{k}$ be the $k$ th-eigenvalue of the second-order linear eigenvalue problem

$$
\begin{gathered}
\Delta^{2} u(t-1)+\lambda u(t)=0, \quad t \in \mathbb{T}_{2} \\
u(a+1)=u(b-1)=0
\end{gathered}
$$

It is well known that $\lambda_{k}$ is simple, and the corresponding eigenfunction

$$
\psi_{k}(t):=\sin \frac{k \pi(t-a-1)}{b-a-2}, \quad t \in \mathbb{T}_{1}, k \in \Lambda
$$

See the study by Kelly and Peterson in [18].

The following result is considered with the spectrum of two-parameter eigenvalue problem:

$$
\begin{aligned}
& \Delta^{4} u(t-2)+\beta \Delta^{2} u(t-1)-\alpha u(t)=0, \quad t \in \mathbb{T}_{2} \\
& u(a+1)=u(b-1)=\Delta^{2} u(a)=\Delta^{2} u(b-2)=0 .
\end{aligned}
$$

It is a slightly generalized version of Shi and Wang [13, Theorem 2.1]. 
Proposition 2.2. $(\alpha, \beta)$ is an eigenvalue pair of (2.15), (2.16) if and only if

$$
\frac{\alpha}{\lambda_{k}^{2}}+\frac{\beta}{\lambda_{k}}=1
$$

for some $k \in \Lambda$.

Proof. Let $r_{1}, r_{2} \in \mathbb{C}$ such that

$$
r_{1}+r_{2}=\beta, \quad r_{1} r_{2}=-\alpha
$$

Define two second-order difference operators $L_{1}, L_{2}: Y \rightarrow Y$ by

$$
\begin{aligned}
& \left(L_{1} y\right)(t):=\Delta^{2} y(t-1)+r_{1} y(t), \quad t \in \mathbb{T}_{2}, \\
& \left(L_{1} y\right)(a+1)=0, \quad\left(L_{1} y\right)(b-1)=0, \\
& \left(L_{2} y\right)(t):=\Delta^{2} y(t-1)+r_{2} y(t), \quad t \in \mathbb{T}_{2}, \\
& \left(L_{2} y\right)(a+1)=0, \quad\left(L_{2} y\right)(b-1)=0 .
\end{aligned}
$$

Then, for $y \in Y$ and $t \in \mathbb{T}_{1}$,

$$
\begin{aligned}
L_{2} \circ L_{1} y(t) & =L_{2}\left(\Delta^{2} y(t-1)+r_{1} y(t)\right) \\
& =\Delta^{2}\left(\Delta^{2} y(t-2)+r_{1} y(t-1)\right)+r_{2}\left(\Delta^{2} y(t-1)+r_{1} y(t)\right) \\
& =\Delta^{4} y(t-2)+\left(r_{1}+r_{2}\right) \Delta^{2} y(t-1)+r_{1} r_{2} y(t) \\
& =\Delta^{4} y(t-2)+\beta \Delta^{2} y(t-1)-\alpha y(t) \\
& =\left(L_{Y}\right) y(t) .
\end{aligned}
$$

We claim that if (2.15), (2.16) possess a nontrivial solution $y$, then either $r_{1}=\lambda_{k}$ or $r_{2}=\lambda_{k}$ for some $k \in \Lambda$. In either case, $\sin (k \pi(t-a-1) /(b-a-2)),\left(t \in \mathbb{T}_{1}\right)$, is a nontrivial solution of (2.15), (2.16).

In fact, if $r_{2} \neq \lambda_{k}$ for all $k \in \Lambda$, then (2.20) implies that

$$
\left(L_{1} y\right)(t)=0, \quad t \in \mathbb{T}_{1}
$$

This is

$$
\begin{gathered}
\Delta^{2} y(t-1)+r_{1} y(t)=0, \quad t \in \mathbb{T}_{2}, \\
y(a+1)=y(b-1)=0 .
\end{gathered}
$$


Thus, $r_{1}=\lambda_{k}$ for some $k \in \Lambda$, and

$$
y(t)=\sin \frac{k \pi(t-a-1)}{b-a-2}, \quad t \in \mathbb{T}_{1} .
$$

If $r_{2}=\lambda_{k}$ for some $k \in \Lambda$, then (2.20) implies that

$$
L_{1} y(t)=\gamma \psi_{k}(t), \quad t \in \mathbb{T}_{1},
$$

for some $\gamma \in \mathbb{R} \backslash\{0\}$. This is

$$
\begin{gathered}
\Delta^{2} y(t-1)+r_{1} y(t)=r \psi_{k}(t), \quad t \in \mathbb{T}_{2}, \\
y(a+1)=y(b-1)=0 .
\end{gathered}
$$

Since $r \neq 0$, it follows that

$$
r_{1} \neq \lambda_{k}, \quad k \in \Lambda
$$

This implies that (2.25), (2.26) have a unique solution

$$
y(t):=\left(L_{1}\right)^{-1} \gamma \psi_{k}(t), \quad t \in \mathbb{T}_{1} .
$$

We show that

$$
r_{1}=\lambda_{k}+\gamma, \quad y(t)=\psi_{k}(t)
$$

In fact, from (2.25) we have

$$
\begin{aligned}
\sum_{t=a+2}^{b-2} \gamma \psi_{k}^{2}(t) & =\sum_{t=a+2}^{b-2}\left(\Delta^{2} y(t-1)+r_{1} y(t)\right) \psi_{k}(t) \\
& =\sum_{t=a+2}^{b-2}\left(\Delta^{2} \psi_{k}(t-1)+r_{1} \psi_{k}(t)\right) y(t) \\
& =\sum_{t=a+2}^{b-2}\left(r_{1}-\lambda_{k}\right) \psi_{k}(t) y(t) \\
& =\sum_{t=a+2}^{b-2}\left(r_{1}-\lambda_{k}\right) \psi_{k}^{2}(t)
\end{aligned}
$$

which implies that $\gamma=r_{1}-\lambda_{k}$, and, subsequently, $y(t)=\psi_{k}(t)$.

Therefore, the claim is true. 
Now, (2.17) follows by substituting this solution into (2.15), (2.16). Reciprocally, if (2.17) holds, then, clearly, $\sin (k \pi(t-a-1) /(b-a-2)),\left(t \in \mathbb{T}_{1}\right)$ is a nontrivial solution of (2.15), (2.16).

Remark 2.3. From the proof of Proposition 2.2, we see that if (2.15) subjects to (1.9), then we can factor $\left.L\right|_{Y}$ as follows:

$$
\left.L\right|_{Y}=L_{2} \circ L_{1}
$$

However, this cannot be done if (2.15) subjects to (1.11). So, (1.9) has more advantage than (1.11) in the study of the spectrum of two-parameter linear eigenvalue problems.

Next, for $j \in \mathbb{N}$, let us set

$$
L_{j}=\left\{(\alpha, \beta) \mid \frac{\alpha}{\lambda_{j}^{2}}+\frac{\beta}{\lambda_{j}}=1\right\} .
$$

In view of the Proposition 2.2, we call $L_{j}$ an eigenline of (2.15), (2.16). We note that an eigenvalue pair $(\alpha, \beta)$ can belong to at most two eigenlines. If $(\alpha, \beta)$ belongs to just one $L_{j}$, then the corresponding eigenspace is spanned by $\sin (k \pi(t-a-1) /(b-a-2))$. If $(\alpha, \beta)$ belongs to $L_{j} \cap L_{k} \quad(j \neq k)$, then the corresponding eigenspace is spanned by $\sin (j \pi(t-a-1) /(b-a-2))$ and $\sin (k \pi(t-a-1) /(b-a-2))$.

Suppose that the pair $(\alpha, \beta)$ is not an eigenvalue pair of $(2.15),(2.16)$, that is,

$$
\frac{\alpha}{\lambda_{k}^{2}}+\frac{\beta}{\lambda_{k}} \neq 1
$$

for all $k \in \Lambda$, and that $h \in Z$ :

$$
h(t)=\sum_{k=1}^{b-a-3} h_{k} \sin \frac{k \pi(t-a-1)}{b-a-2}, \quad t \in \mathbb{T}_{2} .
$$

From the Fredholm Alternative, it follows that the boundary value problem

$$
\begin{gathered}
\Delta^{4} u(t-2)+\beta \Delta^{2} u(t-1)-\alpha u(t)=h(t), \quad t \in \mathbb{T}_{2} \\
u(a+1)=u(b-1)=\Delta^{2} u(a)=\Delta^{2} u(b-2)=0
\end{gathered}
$$

has a unique solution for each $h \in Z$. Moreover, this solution admits a Fourier series expansion of the form

$$
\begin{gathered}
u(t)=\sum_{k=1}^{b-a-3} \frac{h_{k} \sin (k \pi(t-a-1) /(b-a-2))}{\left(\lambda_{k}^{2}-\alpha-\beta \lambda_{k}\right)}, \quad t \in \mathbb{T}_{2,} \\
u(a+1):=0, \quad u(b-1):=0, \quad u(a):=-u(a+2), \quad u(b):=-u(b-2) .
\end{gathered}
$$


Also, we have

$$
\begin{gathered}
\Delta^{2} u(t-1)=-\sum_{k=1}^{b-a-3} \frac{\lambda_{k} h_{k} \sin (k \pi(t-a-1) /(b-a-2))}{\left(\lambda_{k}^{2}-\alpha-\beta \lambda_{k}\right)}, t \in \mathbb{T}_{2}, \\
\Delta^{2} u(a):=0, \quad \Delta^{2} u(b-2):=0 .
\end{gathered}
$$

From (2.36) and (2.37), we can easily see that the operators $A: Z \rightarrow Z, B: Z \rightarrow Z$ defined by

$$
A(h)(t)=u(t), \quad B(h)(t)=\Delta^{2} u(t-1), \quad t \in \mathbb{T}_{2},
$$

are compact linear operators. In (2.38), $u$ is the solution of (2.35), (2.16) corresponding to $h \in Z$. The norms of $A$ and $B$ are, respectively, given by

$$
\|A\|_{Z \rightarrow Z}=\max _{k \in \Lambda}\left\{\frac{1}{\left|\lambda_{k}^{2}-\alpha-\beta \lambda_{k}\right|}\right\}, \quad\|B\|_{Z \rightarrow Z}=\max _{k \in \Lambda}\left\{\frac{\lambda_{k}}{\left|\lambda_{k}^{2}-\alpha-\beta \lambda_{k}\right|}\right\} .
$$

Finally, as an immediate consequence of Proposition 2.2, we have the following.

Proposition 2.4. Let $\gamma$ and $\delta$ be two constants with $(\gamma, \delta) \in[0, \infty) \times[0, \infty)$ and $\gamma+\delta>0$. Then the generalized eigenvalues of problem

$$
\begin{gathered}
\Delta^{4} u(t-2)+\mu\left(\delta \Delta^{2} u(t-1)-\gamma u(t)\right)=0, \quad t \in \mathbb{T}_{2}, \\
u(a+1)=u(b-1)=\Delta^{2} u(a)=\Delta^{2} u(b-2)=0
\end{gathered}
$$

are given by

$$
\mu_{1}(\gamma, \delta)<\mu_{2}(\gamma, \delta)<\cdots<\mu_{b-a-3}(\gamma, \delta)
$$

where

$$
\mu_{k}(\gamma, \delta)=\frac{\lambda_{k}^{2}}{\gamma+\delta \lambda_{k}}, \quad k \in \Lambda
$$

The generalized eigenfunction corresponding to $\mu_{k}(\gamma, \delta)$ is

$$
\psi_{k}(t)=\sin \frac{k \pi(t-a-1)}{b-a-2}, \quad t \in \mathbb{T}_{1}
$$




\section{Existence Results for Nonresonant Problems}

Theorem 3.1. Assume that the pair $(\alpha, \beta)$ satisfies

$$
\frac{\alpha}{\lambda_{k}^{2}}+\frac{\beta}{\lambda_{k}} \neq 1
$$

for all $k \in \Lambda$ and that there are positive constants $a^{*}, b^{*}$, and $c^{*}$ such that

$$
\begin{gathered}
a^{*} \max _{k \in \Lambda}\left\{\frac{1}{\left|\lambda_{k}^{2}-\alpha-\beta \lambda_{k}\right|}\right\}+b^{*} \max _{k \in \Lambda}\left\{\frac{\lambda_{k}}{\left|\lambda_{k}^{2}-\alpha-\beta \lambda_{k}\right|}\right\}<1, \\
|f(t, u, v)-(\alpha u-\beta v)| \leq a^{*}|u|+b^{*}|v|+c^{*}
\end{gathered}
$$

for all $t \in \mathbb{T}_{2}, u, v \in \mathbb{R}$, then (1.6), (1.7) possess at least one solution.

Remark 3.2. It is not difficult to see that (3.1), (3.2) imply that

$$
\frac{a^{*}}{\left|\lambda_{k}^{2}-\alpha-\beta \lambda_{k}\right|}+\frac{b^{*} \lambda_{k}}{\left|\lambda_{k}^{2}-\alpha-\beta \lambda_{k}\right|}<1
$$

for $k \in \Lambda$. It turns out that (3.4) is equivalent to the fact that the square $\left[\alpha-a^{*}, \alpha+a^{*}\right] \times$ $\left[\beta-b^{*}, \beta+b^{*}\right]$ does not intersect any of the eigenlines $L_{j}$ of (2.15), (2.16). From this point of view, (3.1), (3.2) can be thought of as a two-parameter nonresonance condition relative to the eigenlines $L_{j}$.

Proof of Theorem 3.1. It is easy to check that the problem

$$
\begin{gathered}
\Delta^{4} u(t-2)=0, \quad t \in \mathbb{T}_{2}, \\
u(a+1)=r_{1}, \quad u(b-1)=r_{2}, \quad \Delta^{2} u(a)=r_{3}, \quad \Delta^{2} u(b-2)=r_{4}
\end{gathered}
$$

has a unique solution $l(t)$. Set

$$
y(t):=u(t)-l(t), \quad t \in \mathbb{T}_{0} .
$$

Then (1.6),(1.7) can be rewritten as

$$
\begin{gathered}
\Delta^{4} y(t-2)=f\left(t, y(t)+l(t), \Delta^{2}(y(t-1)+l(t-1))\right), \quad t \in \mathbb{T}_{2}, \\
y(a+1)=y(b-1)=\Delta^{2} y(a)=\Delta^{2} y(b-2)=0 .
\end{gathered}
$$


Since

$$
\begin{aligned}
& \left|f\left(t, y(t)+l(t), \Delta^{2}(y(t-1)+l(t-1))\right)-\left[\alpha(y(t)+l(t))-\beta\left(\Delta^{2}(y(t-1)+l(t-1))\right)\right]\right| \\
& \quad \leq a^{*}|y|+b^{*}\left|\Delta^{2} y(t-1)\right|+c^{* *}
\end{aligned}
$$

with

$$
c^{* *}=a^{*} \max _{t \in \mathbb{T}_{0}}|l(t)|+b^{*} \max _{t \in \mathbb{T}_{1}}\left|\Delta^{2} l(t-1)\right|+c^{*},
$$

it follows that (3.2) and (3.3) still hold except that $c^{*}$ is replaced by $c^{* *}$. So, we may suppose that $r_{1}=r_{2}=r_{3}=r_{4}=0$ in (1.7).

Let us define $T: Z \times Z \rightarrow Z \times Z$ by

$$
T(u, v)=(A(f(\cdot, u, v)-(\alpha u-\beta v)), B(f(\cdot, u, v)-(\alpha u-\beta v)))
$$

where $A$ and $B$ are the operators defined in (2.38). The growth condition (3.3) together with the compactness $A$ and $B$ implies that $T$ is a completely continuous operator. By Remark 2.1, the problem

$$
\begin{gathered}
\Delta^{4} u(t-2)=f\left(t, u(t), \Delta^{2} u(t-1)\right), \quad t \in \mathbb{T}_{2}, \\
u(a+1)=u(b-1)=\Delta^{2} u(a)=\Delta^{2} u(b-2)=0
\end{gathered}
$$

is equivalent to the fixed point problem in $Z \times Z$ :

$$
(u, v)=T(u, v)
$$

We will study this fixed point problem by means of the well-known Leray-Schauder principle [18]. To do this, we show that there is a uniform bound independent of $\lambda \in[0,1]$ for the solutions of the equation

$$
(u, v)=\lambda T(u, v)
$$

Thus, let $(u, v)$ be a solution of (3.13). From the definition of $T$ and (3.3), we obtain the result that

$$
\begin{gathered}
\|u\|_{Z} \leq\|A\|_{Z \rightarrow Z}\left\{a\|u\|_{Z}+b\|v\|_{Z}+c\right\} \\
\|v\|_{Z} \leq\|B\|_{Z \rightarrow Z}\left\{a\|u\|_{Z}+b\|v\|_{Z}+c\right\} .
\end{gathered}
$$


Combining (3.14) and (3.15) and using (3.2) and (2.38), we obtain the existence of a constant $M=M\left(a^{*}, b^{*}, c^{*},\|A\|_{Z \rightarrow Z},\|B\|_{Z \rightarrow Z}\right)$ such that

$$
\|u\|_{Z}+\|v\|_{Z} \leq M
$$

By the Leray-Schauder principle [19], we conclude the existence of at least one solution of (3.12), and the theorem follows.

\section{Existence and Multiplicity Results for Perturbations of Resonant Linear Problems}

In this section, we consider the perturbations of resonant linear problems of the form

$$
\begin{gathered}
\Delta^{4} u(t-2)+\left(\mu_{k}+\mu\right)\left(\delta \Delta^{2} u(t-1)-\gamma u(t)\right)=g(t, u(t))+h(t), \quad t \in \mathbb{T}_{2} \\
u(a+1)=u(b-1)=\Delta^{2} u(a)=\Delta^{2} u(b-2)=0
\end{gathered}
$$

where $(\gamma, \delta) \in[0, \infty) \times[0, \infty)$ with $\gamma+\delta>0, \mu_{k}=\mu_{k}(\gamma, \delta)$, and $g$ and $h$ satisfy the following.

(H1) (Sublinear growth condition) $g: \mathbb{T}_{2} \times \mathbb{R} \rightarrow \mathbb{R}$ is continuous, and there exist $\alpha \in[0,1)$, $C_{1}, C_{2} \in(0, \infty)$ such that

$$
|g(t, s)| \leq C_{1}|s|^{\alpha}+C_{2}, \quad s \in \mathbb{R}, t \in \mathbb{T}_{2}
$$

(H2) There exists $\beta>0$ such that

$$
s g(t, s)>0, \quad \text { for } t \in \mathbb{T}_{2},|s|>\beta
$$

(H3) $h: \mathbb{T}_{2} \rightarrow \mathbb{R}$ satisfies

$$
\sum_{t=a+2}^{b-2} h(t) \psi_{k}(t)=0
$$

We will establish some a priori bounds and use these together with Leray-Schauder continuation and bifurcation arguments to reduce results which say that there are multiple solutions of $(4.1)_{\mu^{\prime}}(4.1)$ for $\mu$ on one side of zero and guarantee the existence of at least one solution for $\mu=0$ and $\mu$ on the other side of zero. To wit, we have the following.

Theorem 4.1. Let (H1), (H2), and (H3) hold. Then there exist $\mu_{-}<0<\mu_{+}$such that $(4.1)_{\mu^{\prime}}$ have

(1) at least one solution if $\mu \in\left[0, \mu_{+}\right]$,

(2) at least three solutions if $\mu \in\left[\mu_{-}, 0\right)$. 
We have the following "dual" theorem if (H2) is replaced by the assumption $\left(\mathrm{H}^{\prime}\right)$ that there exists $\beta>0$ such that

$$
s g(t, s)<0, \quad \text { for } t \in \mathbb{T}_{2},|s|>\beta
$$

Theorem 4.2. Let (H1), (H2'), and (H3) hold. Then there exist $\mu_{-}<0<\mu_{+}$such that $(4.1)_{\mu^{\prime}}$ have

(1) at least one solution if $\mu \in\left[\mu_{-}, 0\right]$,

(2) at least three solutions if $\mu \in\left(0, \mu_{+}\right]$.

Define $\mathcal{\perp}: X \rightarrow Z$ by

$$
(\mathcal{L} y)(t)=\Delta^{4} u(t-2)+\mu_{k}\left(\delta \Delta^{2} u(t-1)-\gamma u(t)\right), \quad t \in \mathbb{T}_{2}
$$

Define $F: Z \rightarrow Z$ by

$$
(F u)(t)=g(t, u(t)), \quad t \in \mathbb{T}_{2}
$$

It is easy to check that $F: Z \rightarrow Z$ is continuous. Obviously $(4.1)_{\mu}(4.1)$ are equivalent to

$$
\mathcal{L} u+\mu\left(\delta \Delta^{2} u(t-1)-\gamma u(t)\right)=F(u)+h .
$$

Define an operator $P: X \rightarrow X$ by

$$
(P x)(t)=\psi_{k}(t) \sum_{s=a+2}^{b-2} x(s) \psi_{k}(s), \quad t \in \mathbb{T}_{0},
$$

where

$$
\psi_{k}(a):=-\psi_{k}(a+2), \quad \psi_{k}(b):=-\psi_{k}(b-2)
$$

It is easy to show the following.

Lemma 4.3. $P$ is a projection and $\operatorname{Im}(P)=\operatorname{Ker}(\mathcal{L})$.

Define an operator $E: Z \rightarrow Z$ by

$$
(E y)(t)=y(t)-\psi_{k}(t) \sum_{s=a+2}^{b-2} y(s) \psi_{k}(s), \quad t \in \mathbb{T}_{2}
$$

Obviously, we have the following. 
Lemma 4.4. $E$ is a projection and $\operatorname{Im}(E)=\operatorname{Im}(\mathcal{L})$.

It is clear that

$$
X=X_{P} \oplus X_{I-P}, \quad Z=Z_{I-E} \oplus Z_{E},
$$

where $I$ represents the identity operator and $X_{P}, X_{I-P}, Z_{I-E}$, and $Z_{E}$ are the images of $P, I-P$, $I-E$, and $E$, respectively.

It is obvious that the restriction of $\mathcal{\perp}$ to $X_{I-P}$ is a bijection from $X_{I-P}$ onto $Z_{E}$, the image of $\mathcal{L}$. We define $M: Z_{E} \rightarrow X_{I-P}$ by

$$
M:=\left(\left.\mathcal{L}\right|_{X_{I-P}}\right)^{-1}
$$

Since $\operatorname{ker}(\mathcal{L})=\operatorname{span}\left\{\psi_{k}\right\}$, we see that each $x \in X$ can be uniquely decomposed into

$$
x=\rho \psi_{k}+v
$$

for some $\rho \in \mathbb{R}$, and $v \in X_{I-P}$. For $z \in Z$, we also have the decomposition

$$
z=\tau \psi_{k}+\bar{h}
$$

with $\tau \in \mathbb{R}$ and $\bar{h} \in Z_{E}$.

Lemma 4.5. Equations $(4.1)_{\mu^{\prime}}(4.1)$ are equivalent to the system

$$
\begin{gathered}
\mathcal{L} v+\mu\left(\delta \Delta^{2} v(t-1)-\gamma v(t)\right)=E F\left(\rho \psi_{k}+v\right)+h \\
\mu \sum_{s=a+2}^{b-2}\left(\delta \Delta^{2} \psi_{k}(s-1)-\gamma \psi_{k}(s)\right) \psi_{k}(s)=\sum_{s=a+2}^{b-2} \psi_{k}(s) f\left(s, \rho \psi_{k}(s)+v(s)\right) .
\end{gathered}
$$

Lemma 4.6. Let (H1) and (H2) hold. Then there exists $R_{0}$ such that any solution $y$ of $(4.1)_{\mu}$ satisfies

$$
\|y\|_{X}<R_{0}
$$

as long as

$$
0 \leq \mu \leq \widehat{\delta}:=\frac{1}{2\|M J\|_{X_{I-P} \rightarrow X_{I-P}}},
$$

where $J: X \rightarrow Z$ is defined by

$$
(J x)(t):=\delta \Delta^{2} x(s-1)-\gamma x(s), \quad t \in \mathbb{T}_{2} .
$$


Proof. Obviously $\left.(\mathcal{L}+\mu J)\right|_{X_{I-P}}: X_{I-P} \rightarrow Z_{E}$ is invertible for $|\mu| \leq \widehat{\delta}$. Moreover, by (4.17),

$$
\begin{aligned}
& \left\|\left.(\mathcal{L}+\mu J)\right|_{X_{I-P}} ^{-1}\right\|_{Z_{E} \rightarrow X_{I-P}}=\left\|\left[\left(\left.\mathcal{L}\right|_{X_{I-P}}\right)(I+\mu M J)\right]^{-1}\right\|_{Z_{E} \rightarrow X_{I-P}} \\
& =\left\|[(I+\mu M J)]^{-1}\right\|_{X_{I-P} \rightarrow X_{I-P}}\|M\|_{Z_{E} \rightarrow X_{I-P}} \leq 2\|M\|_{Z_{E} \rightarrow X_{I-P}} .
\end{aligned}
$$

Let $y=\rho \psi_{k}+v$ be any solution of $(4.1)_{\mu^{\prime}}(4.1)$. Then we have that, if $\rho \neq 0$,

$$
\begin{aligned}
\|v\|_{X}= & \left\|\left.(\mathcal{L}+\mu J)\right|_{X_{I-P}} ^{-1} E\left(h-F\left(\rho \psi_{k}+v\right)\right)\right\|_{X} \\
\leq & \left\|\left.(\mathcal{L}+\mu J)\right|_{X_{I-P}} ^{-1}\right\|_{Z_{E} \rightarrow X_{I-P}}\|E\|_{Z \rightarrow Z_{E}}\left[\|h\|_{Z}+C_{1}\left(|\rho|\left\|\psi_{k}\right\|_{Z}+\|v\|_{Z}\right)^{\alpha}+C_{2}\right] \\
\leq & 2\|M\|_{Z_{E} \rightarrow X_{I-P}}\|E\|_{Z \rightarrow Z_{E}}\left[\|h\|_{Z}+C_{1}\left(|\rho|\left\|\psi_{k}\right\|_{Z}+\|v\|_{Z}\right)^{\alpha}+C_{2}\right] \\
\leq & 2\|M\|_{Z_{E} \rightarrow X_{I-P}}\|E\|_{Z \rightarrow Z_{E}}\left[\|h\|_{Z}+C_{1}\left(|\rho|\left\|\psi_{k}\right\|_{X}+\|v\|_{X}\right)^{\alpha}+C_{2}\right] \\
= & 2\|M\|_{Z_{E} \rightarrow X_{I-P}}\|E\|_{Z \rightarrow Z_{E}}\left[\|h\|_{Z}+C_{1}\left(|\rho|\left\|\psi_{k}\right\|_{X}\right)^{\alpha}\left(1+\frac{\|v\|_{X}}{|\rho|\left\|\psi_{k}\right\|_{X}}\right)^{\alpha}+C_{2}\right] \\
\leq & 2\|M\|_{Z_{E} \rightarrow X_{I-P}}\|E\|_{Z \rightarrow Z_{E}}\left[\|h\|_{Z}+C_{1}\left(|\rho|\left\|\psi_{k}\right\|_{X}\right)^{\alpha}\left(1+\frac{\alpha\|v\|_{X}}{|\rho|\left\|\psi_{k}\right\|_{X}}\right)+C_{2}\right] \\
= & 2\|M\|_{Z_{E} \rightarrow X_{I-P}}\|E\|_{Z \rightarrow Z_{E}} \\
& \times\left[\|h\|_{Z}+C_{1}\left(|\rho|\left\|\psi_{k}\right\|_{X}\right)^{\alpha}\left(1+\frac{\alpha}{\left(|\rho|\left\|\psi_{k}\right\|_{X}\right)^{1-\alpha}} \frac{\|v\|_{X}}{\left(|\rho|\left\|\psi_{k}\right\|_{X}\right)^{\alpha}}\right)+C_{2}\right],
\end{aligned}
$$

and hence

$$
\frac{\|v\|_{X}}{\left(|\rho|\left\|\psi_{k}\right\|_{X}\right)^{\alpha}} \leq \frac{C_{3}}{\left(|\rho|\left\|\psi_{k}\right\|_{X}\right)^{\alpha}}+C_{4}+\frac{\alpha C_{4}}{\left(|\rho|\left\|\psi_{k}\right\|_{X}\right)^{1-\alpha}} \frac{\|v\|_{X}}{\left(|\rho|\left\|\psi_{k}\right\|_{X}\right)^{\alpha}}
$$

where

$$
C_{3}=\|M\|_{Z_{E} \rightarrow X_{I-P}}\|E\|_{Z \rightarrow Z_{E}}\left(\|h\|_{Z}+C_{2}\right), \quad C_{4}=2 C_{1}\|M\|_{Z_{E} \rightarrow X_{I-P}}\|E\|_{Z \rightarrow Z_{E}} .
$$

If

$$
|\rho| \geq \frac{\left(2 \alpha C_{4}\right)^{1 /(1-\alpha)}}{\left\|\psi_{k}\right\|_{X}}=: \tilde{C}
$$


then we have

$$
\frac{\|v\|_{X}}{\left(|\rho|\left\|\psi_{k}\right\|_{X}\right)^{\alpha}} \leq \frac{2 C_{3}}{\left(\tilde{C}\left\|\psi_{k}\right\|_{X}\right)^{\alpha}}+2 C_{4}=: C^{*} .
$$

If we assume that the conclusion of the lemma is false, we obtain a sequence $\left\{\eta_{n}\right\}$ with $0 \leq \eta_{n} \leq \widehat{\delta}$ and $\eta_{n} \rightarrow 0$, and a sequence of corresponding solutions $\left\{y_{n}=\rho_{n} \psi_{k}+v_{n}\right\}$ of $(4.1)_{\eta_{n}}$ (4.1) such that $\left\|y_{n}\right\|_{X} \rightarrow+\infty$. From (4.24), we conclude that it is necessary that $\left|\rho_{n}\right| \rightarrow+\infty$. We may assume that

$$
\rho_{n} \longrightarrow+\infty, \quad \rho_{n} \geq \tilde{C} \quad \forall n \in \mathbb{N}
$$

since the other case can be treated by the same way. Thus (4.24) yields that

$$
\left\|v_{n}\right\|_{X}: \leq \widehat{C}\left|\rho_{n}\right|^{\alpha}
$$

with $\widehat{C}:=C^{*}\left\|\psi_{k}\right\|_{X}^{\alpha}$.

Now from (4.15), we get that

$$
\rho_{n} \eta_{n} \sum_{s=a+2}^{b-2}\left(\delta \Delta^{2} \psi_{k}(s-1)-\gamma \psi_{k}(s)\right) \psi_{k}(s)=\sum_{s=a+2}^{b-2} \psi_{k}(s) f\left(s, \rho_{n} \psi_{k}(s)+v_{n}(s)\right) .
$$

By (4.17) and (4.27), it follows that

$$
\sum_{s=a+2}^{b-2} \psi_{k}(s) f\left(s, \rho_{n} \psi_{k}(s)+v_{n}(s)\right) \leq 0 .
$$

Let

$$
\begin{aligned}
& A^{+}=\left\{t \mid t \in\{a+2, \ldots, b-2\}, \psi_{k}(t)>0\right\}, \\
& A^{-}=\left\{t \mid t \in\{a+2, \ldots, b-2\}, \psi_{k}(t)<0\right\} .
\end{aligned}
$$

It is easy to see that

$$
A^{+} \cup A^{-} \neq \emptyset, \quad \min \left\{\left|\psi_{k}(t)\right| \mid t \in A^{+} \cup A^{-}\right\}>0 .
$$

Combining (4.30) and (4.26), we conclude that there exists a positive constant $\Gamma$ such that, for $n \in \mathbb{N}$,

$$
\left\|v_{n}\right\|_{X} \leq \Gamma\left(\left|\rho_{n}\right| \min \left\{\left|\psi_{k}(t)\right| \mid t \in A^{+} \cup A^{-}\right\}\right)^{\alpha},
$$


which implies that

$$
\begin{aligned}
& \lim _{\rho_{n} \rightarrow+\infty} \min \left\{\rho_{n} \psi_{k}(t)+v_{n}(t) \mid t \in A^{+}\right\}=+\infty, \\
& \lim _{\rho_{n} \rightarrow+\infty} \min \left\{\rho_{n} \psi_{k}(t)+v_{n}(t) \mid t \in A^{-}\right\}=-\infty .
\end{aligned}
$$

Applying (4.32), (4.30), and (H2), we conclude that

$$
\begin{aligned}
& \sum_{s=a+2}^{b-2} \psi_{k}(s) f\left(s, \rho_{n} \psi_{k}(s)+v_{n}(s)\right) \\
& \quad=\sum_{s \in A^{+}} \psi_{k}(s) f\left(s, \rho_{n} \psi_{k}(s)+v_{n}(s)\right)+\sum_{s \in A^{-}} \psi_{k}(s) f\left(s, \rho_{n} \psi_{k}(s)+v_{n}(s)\right)>0,
\end{aligned}
$$

which contracts (4.28).

Using the similar arguments, we may establish the following lemma.

Lemma 4.7. Let (H1) and (H2') hold. Then there exists $R_{0}$ such that any solution y of $(4.1)_{\mu^{\prime}}(4.1)$ satisfies

$$
\|y\|_{X}<R_{0}
$$

as long as

$$
-\widehat{\delta} \leq \mu \leq 0
$$

where $\widehat{\delta}$ is given in (4.17).

Lemma 4.8. Let (H1) and (H2) hold. Then there exists $R_{1}: R_{1} \geq R_{0}$ such that, for $0 \leq \mu \leq \widehat{\delta}$ and $R \geq R_{1}$, one has

$$
\operatorname{deg}(j \circ(\mathcal{L}+\mu J-F-h), B(R), 0)=\operatorname{deg}(j \circ(\mathcal{L}+\widehat{\delta} J), B(R), 0)= \pm 1,
$$

where $j: Z \rightarrow X$ is the natural homomorphism, $B(R)=\left\{u \in X\|u\|_{X}<R\right\}$, and "deg" denotes Leray-Schauder degree when $\mu \neq 0$ and coincidence degree when $\mu=0$ (see the study by Gaines and Mawhin in [20]). Therefore (4.7) $\mu$ has a solution in $\bar{B}(R)$ for $\mu \in[0, \widehat{\delta}]$.

Proof. By Lemma 4.6 and the definition of $\mathcal{L}$, the degree

$$
\operatorname{deg}(j \circ(\mathcal{L}+\mu J-F-h), B(R), 0)
$$

is well defined for $\mu \in[0, \widehat{\delta}]$ and is a constant with respect to $\mu$. 
Discrete Dynamics in Nature and Society

Now if $(\mu, y) \in[0,1] \times X$ is a solution of

$$
\mathcal{L} y+\widehat{\delta}\left(\delta \Delta^{2} y(s-1)-\gamma y(s)\right)-\mu(F(y)+h)=0,
$$

then we have

$$
\|y\|_{X}=\mu\left\|(\mathcal{L}+\widehat{\delta} J)^{-1}(h+F(y))\right\|_{X} \leq\left\|(\mathcal{L}+\widehat{\delta} J)^{-1}\right\|_{Z_{E} \rightarrow X_{I-P}}\left(\|h\|_{Z}+C_{1}\|y\|_{X}^{\alpha}+C_{2}\right) .
$$

Hence there exists $R_{0}^{\prime}>0$ such that $\|y\|_{X}<R_{0}^{\prime}$. Thus if $R_{1}=\max \left\{R_{0}^{\prime}, R_{0}\right\}$, then we have, whenever $R>R_{1}$, that

$$
\operatorname{deg}(j \circ(\mathcal{L}+\widehat{\delta} J-F-h), B(R), 0)=\operatorname{deg}(j \circ(\mathcal{L}+\widehat{\delta} J), B(R), 0)= \pm 1,
$$

which completes the proof.

By a similar manner we may establish the following.

Lemma 4.9. Let (H1) and (H2') hold. Then there exists $R_{1}: R_{1} \geq R_{0}$ such that, for $0 \geq \mu \geq-\widehat{\delta}$ and $R \geq R_{1}$, one has

$$
\operatorname{deg}(j \circ(\mathcal{L}+\mu J-F-h), B(R), 0)=\operatorname{deg}(j \circ(\mathcal{L}-\widehat{\delta} J), B(R), 0)= \pm 1 .
$$

Therefore $(4.7)_{\mu}$ has a solution in $\bar{B}(R)$ for $\mu \in[-\widehat{\delta}, 0]$.

Lemma 4.10. Let (H1) and (H2) hold. Then there exists $\delta_{1}>0$ such that, for $-\delta_{1}<\mu<0$, one has

$$
\operatorname{deg}\left(j \circ(\mathcal{L}+\mu J-F-h), B\left(R_{1}\right), 0\right)=\operatorname{deg}\left(j \circ\left(\mathcal{L}+\delta_{1} J\right), B\left(R_{1}\right), 0\right)= \pm 1 .
$$

Proof. Let

$$
\tau_{0}=\inf _{x \in \partial B\left(R_{1}\right) \cap X}\|j \circ(\mathcal{L} x-F(x)-h)\|_{X} .
$$

Then it is not difficult to check that $\tau_{0}>0$. Hence if we take $\delta_{1}$ so small that $\delta_{1} R_{1}<\tau_{0}$, then for $\mu \in\left[-\delta_{1}, \delta_{1}\right]$,

$$
\operatorname{deg}\left(j \circ(\mathcal{L}+\mu J-F-h), B\left(R_{1}\right), 0\right)=\operatorname{deg}\left(j \circ(\mathcal{L}-F-h), B\left(R_{1}\right), 0\right)= \pm 1 .
$$

Lemma 4.11. Let (H1) and (H2') hold. Then there exists $\delta_{1}>0$ such that, for $0 \leq \mu \leq \delta_{1}$, one has

$$
\operatorname{deg}(j \circ(\mathcal{L}+\mu J-F-h), B(R), 0)=\operatorname{deg}\left(j \circ\left(\mathcal{L}-\delta_{1} J\right), B(R), 0\right)= \pm 1 .
$$


Proof of Theorem 4.1. By the study of Massabò and Pejsachowicz in [21, Theorem 1.1], (4.7) ${ }_{\mu}$ has a continuum $\mathcal{C}_{*}=\left\{\left(\mu, y_{\mu}\right)\right\}$ of solutions with $\left\|y_{\mu}\right\|_{X}<R_{1}$ and $\mu \in\left[-\delta_{1}, \widehat{\delta}\right]$. On the other hand, since $F$ is $\mathcal{L}$-completely continuous and satisfies (H1) and since $\mu=0$ is a simple eigenvalue, it follows from the study by Rabinowitz in [22, Theorem 1.6] that $\mu=0$ is a bifurcation point from infinity for $(4.7)_{\mu}$. Moreover, there exist two continua

$$
\mathcal{C}_{\infty}^{ \pm}=\left\{\left(\mu, y_{\mu}\right)\right\} \subset \mathbb{R} \times X
$$

of solutions of $(4.7)_{\mu}$, bifurcating from infinity at $\mu=0$, that is, there exists $\epsilon_{0} \in\left(0,1 / R_{1}\right)$, such that for all $\epsilon: 0<\epsilon \leq \epsilon_{0}$ there exist two continua $\mathcal{C}_{\epsilon}^{+}$and $\mathcal{C}_{\epsilon}^{-}$with

$$
\begin{gathered}
\mathcal{C}_{\epsilon}^{+} \subset \mathcal{C}_{\infty}^{+}, \quad \mathcal{C}_{\epsilon}^{-} \subset \mathcal{C}_{\infty}^{-}, \\
\mathcal{C}_{\epsilon}^{ \pm} \subset\left\{\left(\mu, y_{\mu}\right):\left\|y_{\mu}\right\|_{X} \geq \frac{1}{\epsilon},|\mu|<\epsilon\right\}=: U_{\epsilon}(0, \infty),
\end{gathered}
$$

and $\mathcal{C}_{\epsilon}^{ \pm}$connects $(0, \infty)$ to $\partial U_{\epsilon}(0, \infty)$. Notice that $(\mu, y) \in \mathcal{C}_{\epsilon}^{+}$implies that $y(a+2)>0$, and $(\mu, y) \in \mathcal{C}_{\epsilon}^{-}$implies that $y(a+2)<0$. So,

$$
\mathcal{C}_{\epsilon}^{+} \cap \mathcal{C}_{\epsilon}^{-}=\emptyset
$$

Now, Lemma 4.6 implies that

$$
\mathcal{C}_{\epsilon}^{ \pm} \subseteq\left\{\left(\mu, y_{\mu}\right) \mid\left\|y_{\mu}\right\|_{X} \geq \frac{1}{\epsilon},-\epsilon<\mu<0\right\}
$$

This completes the proof.

Proof of Theorem 4.2. Using similar arguments, we may get the desired results.

\section{Acknowledgments}

The authors are very grateful to the anonymous referees for their valuable suggestions. This work was supported by the NSFC (no. 10671158), NWNU-KJCXGC-03-47, the Spring-sun program (no. Z2004-1-62033), SRFDP (no. 20060736001), and the SRF for ROCS, SEM (2006 [311]).

\section{References}

[1] A. R. Aftabizadeh, "Existence and uniqueness theorems for fourth-order boundary value problems," Journal of Mathematical Analysis and Applications, vol. 116, no. 2, pp. 415-426, 1986.

[2] C. P. Gupta, "Existence and uniqueness theorems for the bending of an elastic beam equation," Applicable Analysis, vol. 26, no. 4, pp. 289-304, 1988.

[3] Y. S. Yang, "Fourth-order two-point boundary value problems," Proceedings of the American Mathematical Society, vol. 104, no. 1, pp. 175-180, 1988. 
[4] M. A. Del Pino and R. F. Manásevich, "Existence for a fourth-order boundary value problem under a two-parameter nonresonance condition," Proceedings of the American Mathematical Society, vol. 112, no. 1 , pp. 81-86, 1991.

[5] D. Franco, D. O’Regan, and J. Perán, "Fourth-order problems with nonlinear boundary conditions," Journal of Computational and Applied Mathematics, vol. 174, no. 2, pp. 315-327, 2005.

[6] R. Ma and H. Wang, "On the existence of positive solutions of fourth-order ordinary differential equations," Applicable Analysis, vol. 59, no. 1-4, pp. 225-231, 1995.

[7] R. Ma, J. Zhang, and S. Fu, "The method of lower and upper solutions for fourth-order two-point boundary value problems," Journal of Mathematical Analysis and Applications, vol. 215, no. 2, pp. 415422, 1997.

[8] B. P. Rynne, "Infinitely many solutions of superlinear fourth order boundary value problems," Topological Methods in Nonlinear Analysis, vol. 19, no. 2, pp. 303-312, 2002.

[9] Z. Bai and H. Wang, "On positive solutions of some nonlinear fourth-order beam equations," Journal of Mathematical Analysis and Applications, vol. 270, no. 2, pp. 357-368, 2002.

[10] Q. Yao, "Existence, multiplicity and infinite solvability of positive solutions to a nonlinear fourthorder periodic boundary value problem," Nonlinear Analysis: Theory, Methods E Applications, vol. 63, no. 2, pp. 237-246, 2005.

[11] Y. Li, "Positive solutions of fourth-order periodic boundary value problems," Nonlinear Analysis: Theory, Methods E Applications, vol. 54, no. 6, pp. 1069-1078, 2003.

[12] R. Ma, "Existence of positive solutions of a fourth-order boundary value problem," Applied Mathematics and Computation, vol. 168, no. 2, pp. 1219-1231, 2005.

[13] G. Shi and Y. Wang, "The eigenvalues and existence of solutions of BVPs for fourth order difference equations," Dynamics of Continuous, Discrete E Impulsive Systems. Series A, vol. 14, no. 5, pp. 631-639, 2007.

[14] R. P. Agarwal and P. J. Y. Wong, Advanced Topics in Difference Equations, vol. 404 of Mathematics and Its Applications, Kluwer Academic Publishers, Dordrecht, The Netherlands, 1997.

[15] J. Henderson, "Positive solutions for nonlinear difference equations," Nonlinear Studies, vol. 4, no. 1, pp. 29-36, 1997.

[16] Z. He and J. Yu, "On the existence of positive solutions of fourth-order difference equations," Applied Mathematics and Computation, vol. 161, no. 1, pp. 139-148, 2005.

[17] B. Zhang, L. Kong, Y. Sun, and X. Deng, "Existence of positive solutions for BVPs of fourth-order difference equations," Applied Mathematics and Computation, vol. 131, no. 2-3, pp. 583-591, 2002.

[18] W. G. Kelley and A. C. Peterson, Difference Equations: An Introduction with Application, Harcourt/Academic Press, San Diego, Calif, USA, 2nd edition, 2001.

[19] E. Zeidler, Nonlinear Functional Analysis and Its Applications. I. Fixed-Point Theorems, Springer, New York, NY, USA, 1986.

[20] R. E. Gaines and J. L. Mawhin, Coincidence Degree, and Nonlinear Differential Equations, vol. 568 of Lecture Notes in Mathematics, Springer, Berlin, Germany, 1977.

[21] I. Massabò and J. Pejsachowicz, "On the connectivity properties of the solution set of parametrized families of compact vector fields," Journal of Functional Analysis, vol. 59, no. 2, pp. 151-166, 1984.

[22] P. H. Rabinowitz, "On bifurcation from infinity," Journal of Differential Equations, vol. 14, pp. 462-475, 1973. 


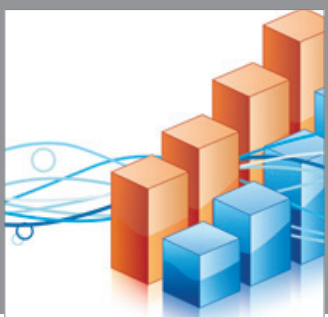

Advances in

Operations Research

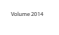

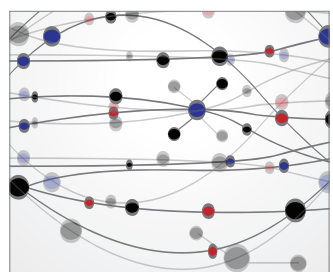

\section{The Scientific} World Journal
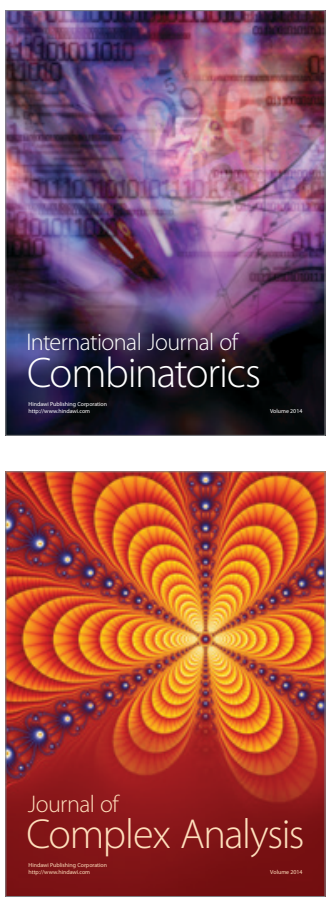

International Journal of

Mathematics and

Mathematical

Sciences
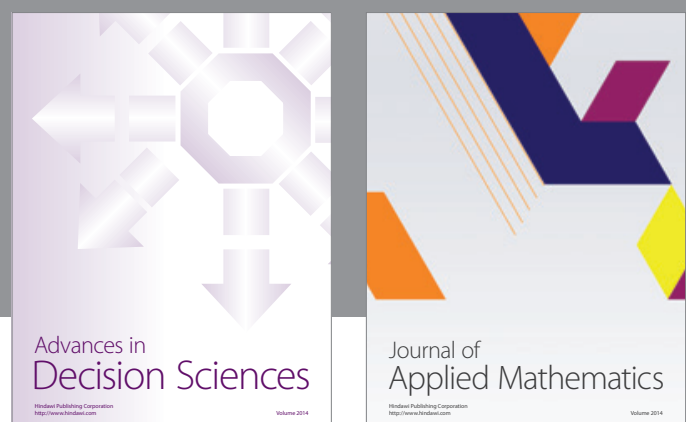

Journal of

Applied Mathematics
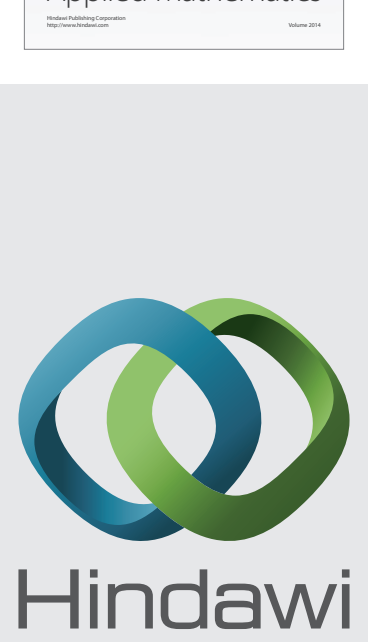

Submit your manuscripts at http://www.hindawi.com
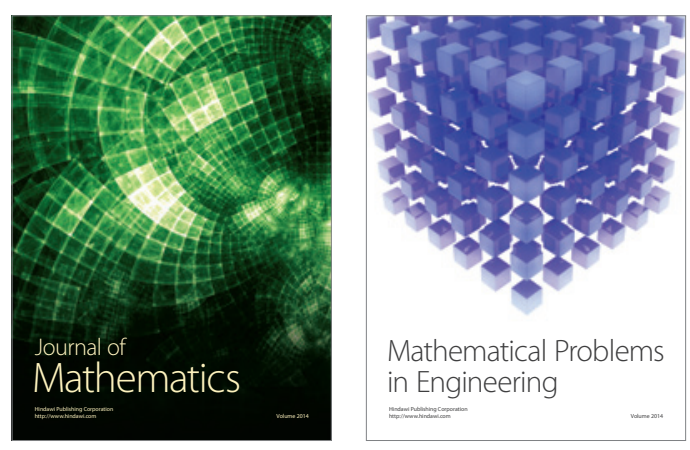

Mathematical Problems in Engineering
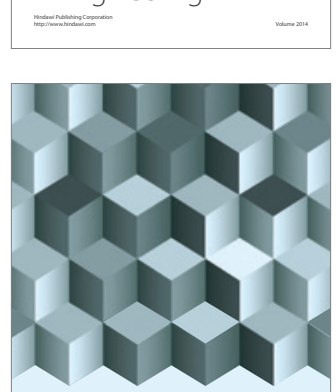

Journal of

Function Spaces
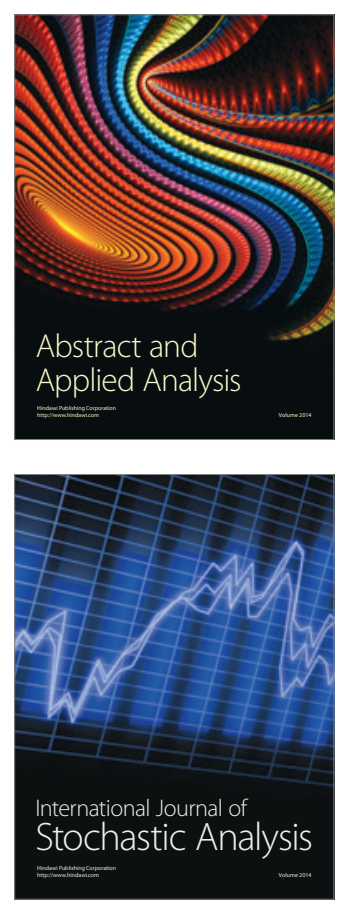

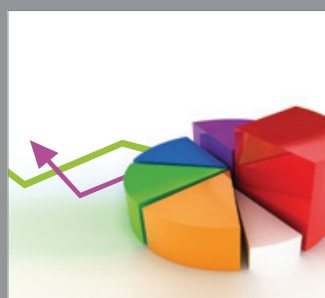

ournal of

Probability and Statistics

Promensencen
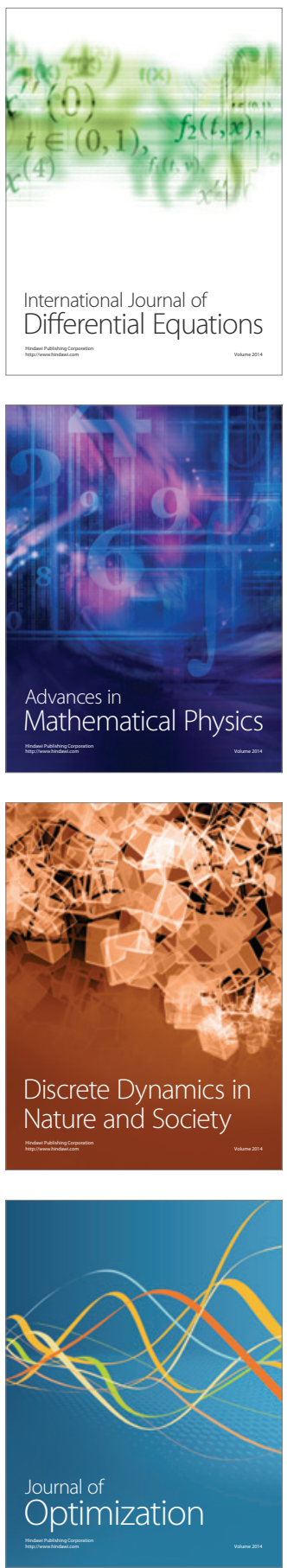